\title{
Improved hyper-reduction approach for the forced vibration analysis of rotating components
}

\author{
Seung-Hoon Kang ${ }^{1} \cdot$ Yongse $\mathrm{Kim}^{1} \cdot$ Haeseong $\mathrm{Cho}^{2} \cdot$ SangJoon Shin ${ }^{1,3}$
}

Received: 29 October 2021 / Accepted: 24 January 2022 / Published online: 18 February 2022

(c) The Author(s) 2022

\begin{abstract}
Forced vibration analysis is an indispensable process for the design of a rotating component. However, rather expensive nonlinear static and linear frequency response analyses are usually accompanied by a frequency domain analysis. The traditional mode-superposition method (MSM) effectively reduces the cost of the frequency response analysis. However, the nonlinear static analysis of earlier processes remains as the computational bottleneck. In this paper, the application of the hyper-reduction method will be proposed along with the model order reduction (MOR) framework for rotating component forced vibration analysis. The energy-conserving sampling and weighting (ECSW) method will be employed for the nonlinear iterative computation. The pre-stressed stiffness matrix of the reduced finite elements (FEs) resulting from the ECSW will be used for the post computation stage. Also, a variety of MOR will be attempted for the performance comparison, including MSM, proper orthogonal decomposition (POD)-based reduced order model (ROM), and a hybrid approach. It is found that the present ECSW-combined MOR will significantly relieve the computational bottleneck, provide a minimal loss of accuracy, and be compatible with both nonlinear and linear analyses of the rotating component forced vibration analysis.
\end{abstract}

Keywords Forced vibration analysis $\cdot$ Model order reduction $\cdot$ Rotating component $\cdot$ Hyper-reduction method

\section{Introduction}

Rotating components including turbine blades and propellers are efficient power delivery apparatus that are acted upon by unsteady aerodynamic forces. As a result of the rotation, the machinery suffers from various vibratory loads that may cause catastrophic failures such as high cycle fatigue (HCF) [1]. Therefore, the design process of the rotating components

SangJoon Shin

ssjoon@snu.ac.kr

Seung-Hoon Kang

shkang94@snu.ac.kr

Yongse Kim

kys0216@snu.ac.kr

Haeseong Cho

hcho@jbnu.ac.kr

1 Department of Aerospace Engineering, Seoul National University, Seoul, 08226, Republic of Korea

2 Department of Aerospace Engineering, Jeonbuk National University, Jeonju, 54896, Republic of Korea

3 Institute of Advanced Aerospace Technology, Seoul National University, Seoul, 08226, Republic of Korea should include an investigation of their vibration characteristics. In this regard, forced vibration analysis examines the structural response to an external vibratory force. To obtain the steady-state solution, the analysis is conducted in a time-harmonic field in which the structural displacements are assumed to vary at the same frequencies as the sinusoidal external load [2].

The computational cost of a frequency response analysis increases with both the number of degrees of freedom (DOFs) and the width of the frequency band. In this respect, the projection-based reduced-order model (ROM) is a favored alternative to reduce the computational costs. Traditionally, a ROM is obtained by the mode superposition method (MSM), which utilizes the eigenmodes for the reduced-order basis [3]. Although MSM reduces the dimension of the full-order model (FOM) dramatically, the modal analysis of the generalized eigenvalue problem on FOM is a prerequisite.

Consideration of the stress-stiffening and spin-softening effect is another computational challenge for the forced vibration analysis. Under the action of the centrifugal force, the component will experience large deformation and corresponding geometric stiffening by the stress state. Also, the centrifugal force increase caused by the structural elongation 
should be included by an equivalent stiffness decrease [4]. Consideration of this stiffness-varying effect usually entails a nonlinear analysis of FOM by an iterative solution procedure, and precedes the aforementioned modal and frequency response analysis.

To reduce the modal and nonlinear analysis cost for rotating components, the reduced-order basis by the proper orthogonal decomposition (POD) is introduced for both the forced vibration analysis procedures $[5,6]$ and for the nonlinear time-transient analysis [7]. POD [8] has received attention for its successful application to various fields using linear and nonlinear formulations [9-11]. The advent of the hyper-reduction method [12-14] alleviated the computational bottleneck induced by the matrix multiplication between the reduced-order basis and FOM equations (i.e. Galerkin projections). Accelerated performance of the hyper-reduction method was obtained for the (pseudo) timetransient analysis in which a repeated Galerkin projection was required for each iteration [15]. Successful application of the hyper-reduction method on the rotating component was found in Ref. [7], where the computational speed for the nonlinear time-transient analysis on the propeller blade became ten to twenty times faster than that of FOM. However, a relatively small number of POD-applications were attempted for the frequency domain analysis of the rotating component.

Kim et al. [5] applied POD to the modal analysis of the rotating component. It was observed that accurate natural frequencies and corresponding modes were obtained for various rotating speeds, while the computational time was reduced. Kang et al. [6] extended the POD-based MOR to an integrated numerical procedure for the rotating component forced vibration analysis. This included the following: nonlinear analysis for the evaluation of the stress-stiffening and spin-softening effect, modal analysis prior to MSM, and forced response analysis in the frequency domain. Regardless of the rotating speed, robust performance was obtained for the given POD basis used. Although efficient results were obtained for the overall numerical analysis, it was observed that non-negligible cost remained in the nonlinear static analysis in which repetitive Galerkin projections were required by Newton-Raphson iteration.

In this paper, an application of the hyper-reduction method on the forced vibration analysis of rotating components will be investigated. The application aims to reduce the computational cost of POD-based ROM by an iterative solution procedure. Then, performance evaluation of various projection-based MOR methods will be conducted including convectional MSM, POD-based ROM, hyper-reduction method and their hybrid approach. The procedure of the forced vibration analysis will include the nonlinear static solution for the evaluation of the stiffness variation by the centrifugal force and time-harmonic solution to obtain the steady-state frequency response. Two applications will be executed in terms of the rotating speed. The first application considers the propeller blade of a slender shape with significant geometrical nonlinearity. The subsequent application concerns a turbine blade composed of a large number of DOFs.

\section{Problem statement}

In this section, equations of the forced vibration analysis will be introduced along with the validation of the FOM by comparison against the commercial software. The finite element (FE) method will be employed and the results of FOM will be compared with those obtained by ANSYS Mechanical [16].

\subsection{Equation of the motion for the nonlinear static analysis}

The first equation of the motion pertains to a nonlinear static analysis for the stress-stiffened and spin-softened stiffness. The governing equations are expressed as follows:

$\mathbf{f}^{\text {int }}(\mathbf{u}(\mu) ; \mu)=\mathbf{f}^{\text {ext }}(\mathbf{u}(\mu) ; \mu)$

where $\mathbf{f}^{\text {int }} \in \mathbb{R}^{N}$, f $\mathbf{f}^{\text {ext }} \in \mathbb{R}^{N}$ are the external and internal force vectors, respectively. $\mathbf{u} \in \mathbb{R}^{N}$ is the displacement vector. $N$ indicates the number of DOFs in FOM. $\mu$ is a parameter. In this paper, $\mu$ will be selected as the rotating speed $\Omega_{\text {rot }}$, which will be crucial for the centrifugal effect and succeeding structural behavior of the rotating component.

The Newton-Raphson algorithm is the conventional iterative approach for the nonlinear solution of Eq. 1. By Taylor's expansion, the relevant linearized form will be obtained as Eq. 2.

$$
\begin{aligned}
\mathbf{K}\left(\mathbf{u}_{k}(\mu) ; \mu\right) \Delta \mathbf{u}_{k}(\mu) & =\mathbf{f}^{\text {ext }}\left(\mathbf{u}_{k}(\mu) ; \mu\right) \\
& -\mathbf{f}^{\text {int }}\left(\mathbf{u}_{k}(\mu) ; \mu\right)
\end{aligned}
$$

$\mathbf{K} \in \mathbb{R}^{N \times N}$ is the stiffness (Jacobian) matrix, and the subscript $k$ indicates an iteration index. $\Delta \mathbf{u}_{k}=\mathbf{u}_{k+1}-\mathbf{u}_{k}$ denotes the displacement increment toward the next iteration step.

For the rotating component, external force $\mathbf{f}^{\text {ext }}$ is provided by the centrifugal force, and the stiffness matrix $\mathbf{K}$ reflects centrifugal effects such as the stress-stiffening and spin-softening. $\mathbf{f}^{\text {int }}, \mathbf{f}^{\text {ext }}$, and $\mathbf{K}$ will be written as Eqs. 3-5, respectively $[3,4,7]$.

$$
\begin{aligned}
& \mathbf{f}^{\mathrm{ext}}\left(\mathbf{u}_{k}(\mu) ; \mu\right)=\mathbf{f}_{0}^{\mathrm{ext}}(\mu)+\mathbf{K}^{\mathrm{sp}}(\mu) \mathbf{u}_{k}(\mu) \\
& \mathbf{K}^{\mathrm{sp}}(\mu)=\sum_{e \in \mathbf{E}} \mathbf{L}_{e}^{T} \mathbf{K}_{e}^{\mathrm{sp}}(\mu) \mathbf{L}_{e}
\end{aligned}
$$




$$
\mathbf{f}^{\text {int }}\left(\mathbf{u}_{k}(\mu) ; \mu\right)=\sum_{e \in \mathbf{E}} \mathbf{L}_{e}^{T} \mathbf{f}_{e}^{\text {int }}\left(\mathbf{L}_{e} \mathbf{u}_{k}(\mu) ; \mu\right)
$$

$$
\begin{aligned}
& \mathbf{K}\left(\mathbf{u}_{k}(\mu) ; \mu\right)=\sum_{e \in \mathbf{E}} \mathbf{L}_{e}^{T} \mathbf{K}_{e}\left(\mathbf{L}_{e} \mathbf{u}_{k}(\mu) ; \mu\right) \mathbf{L}_{e} \\
& \mathbf{K}_{e}\left(\mathbf{L}_{e} \mathbf{u}_{k}(\mu) ; \mu\right)=\mathbf{K}_{e}^{1}\left(\mathbf{L}_{e} \mathbf{u}_{k}(\mu) ; \mu\right) \\
& \quad+\mathbf{K}_{e}^{\mathrm{nl}}\left(\mathbf{L}_{e} \mathbf{u}_{k}(\mu) ; \mu\right)-\mathbf{K}_{e}^{\mathrm{sp}}(\mu)
\end{aligned}
$$

$\mathbf{f}_{0}^{\text {ext }} \in \mathbb{R}^{N}$ is the centrifugal force defined by an initial configuration. $\mathbf{E}=\left\{e_{1}, e_{2}, \ldots, e_{N_{e}}\right\}$ is the set of the element indices, where $N_{e}$ is the total number of the finite elements. $\mathbf{L}_{e} \in \mathbb{R}^{n_{u}^{e} \times N}$ is a Boolean matrix that extracts the contributions of the element $e$, and $n_{u}^{e}$ indicates the number of the displacement DOFs of the element $e . \mathbf{K}^{\mathrm{sp}} \in \mathbb{R}^{N \times N}$ and $\mathbf{K}_{e}^{\mathrm{sp}} \in \mathbb{R}^{n_{u}^{e} \times n_{u}^{e}}$ are the spin-softening matrix and that for the $e$-th element, respectively. $\mathbf{f}_{e}^{\text {int }} \in \mathbb{R}^{n_{u}^{e}}$ and $\mathbf{K}^{e} \in \mathbb{R}^{n_{u}^{e} \times n_{u}^{e}}$ are the $e$-th elemental internal force and stiffness matrix. $\mathbf{K}_{e}^{1} \in \mathbb{R}^{n_{u}^{e} \times n_{u}^{e}}$ and $\mathbf{K}_{e}^{\mathrm{nl}} \in \mathbb{R}^{n_{u}^{e} \times n_{u}^{e}}$ are the $e$-th elemental stiffness matrices of linear and nonlinear strains. In this paper, the vectors and matrices of Eqs. 3-5 will be obtained by updated Lagrangian formulation. After the nonlinear analysis converges, the stiffness matrix $\mathbf{K}\left(\mathbf{u}_{m}(\mu) ; \mu\right)$ will be utilized for the next step, where $m$ indicates the last Newton-Raphson iteration number.

\subsection{Equation of the motion for the frequency response analysis}

Prior to the explanation of the equation for the frequency response analysis, that of the linear time-transient analysis will be introduced as Eq. 6 .

$$
\begin{aligned}
& \mathbf{M}(\mu) \ddot{\mathbf{u}}(t ; \mu)+\mathbf{C}(\mu) \dot{\mathbf{u}}(t ; \mu) \\
& +\mathbf{K}\left(\mathbf{u}_{m}(\mu) ; \mu\right) \mathbf{u}(t ; \mu)=\mathbf{f}^{\text {ext }}(t ; \mu)
\end{aligned}
$$

$\mathbf{M} \in \mathbb{R}^{N \times N}$ and $\mathbf{C} \in \mathbb{R}^{N \times N}$ are the mass and damping matrices. $t$ is time. It is noted that the pre-stressed stiffness matrix $\mathbf{K}$ is obtained from the nonlinear static analysis in Eq. 5, where $\mathbf{u}_{m}$ is the converged displacement vector by Newton-Raphson iteration.

The governing equation for the frequency response analysis assumes that the entire structural component oscillates at a frequency equal to that of the imposed harmonic force. Then, the linear structural dynamic equation will be expressed as Eq. 7.

$$
\begin{aligned}
(- & \Omega^{2} \mathbf{M}(\mu)+j \Omega \mathbf{C}(\mu) \\
& \left.+\mathbf{K}\left(\mathbf{u}_{m}(\mu) ; \mu\right)\right) \mathbf{u}_{c}(\mu ; \Omega)=\mathbf{f}_{c}(\mu)
\end{aligned}
$$

$j$ and $\Omega$ are the unit imaginary number and angular velocity of the excitation force, respectively. $\mathbf{u}_{c} \in \mathbb{C}^{N}$ and $\mathbf{f}_{c} \in \mathbb{C}^{N}$ are the complex displacement and imposed force vector, in which $\operatorname{Re}\left(\mathbf{u}_{c} e^{j \Omega t}\right)$ and $\operatorname{Re}\left(\mathbf{f}_{c} e^{j \Omega t}\right)$ implies the dynamic displacement and force vectors with the harmonic assumption.

\subsection{Validation of the full-order model}

Prior to MOR application, FOM will be validated against the results by ANSYS [16]. The present in-house FOM solver is written in FORTRAN 90 with the Intel Math Kernel Library (MKL) [17]. The validation object is a 54H60 propeller blade, which has a slender configuration that is associated with geometrical nonlinearity. As shown in Fig. 1, the blade is discretized into 10-node tetrahedral elements with $N_{e}=24,151$, and the corresponding number of the displacement DOFs is $N=133,857$ from the ANSYS mesh generator [18]. The material properties of a 7000-series aluminum alloy are considered [19] with Rayleigh-type viscous damping of a mass matrix multiplier of $50 \mathrm{~s}^{-1}$.

For rigorous verification, each analysis module is validated separately. In this subsection, the pre-stressed stiffness matrix of the nonlinear static analysis will not be used for

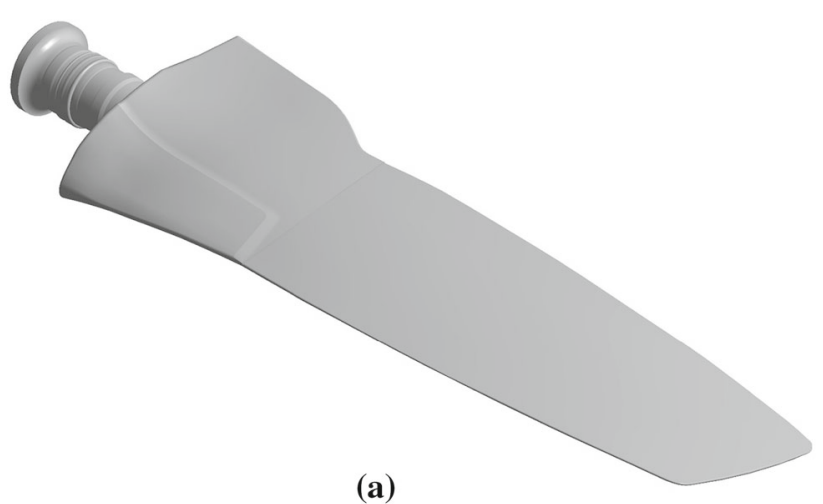

(a)

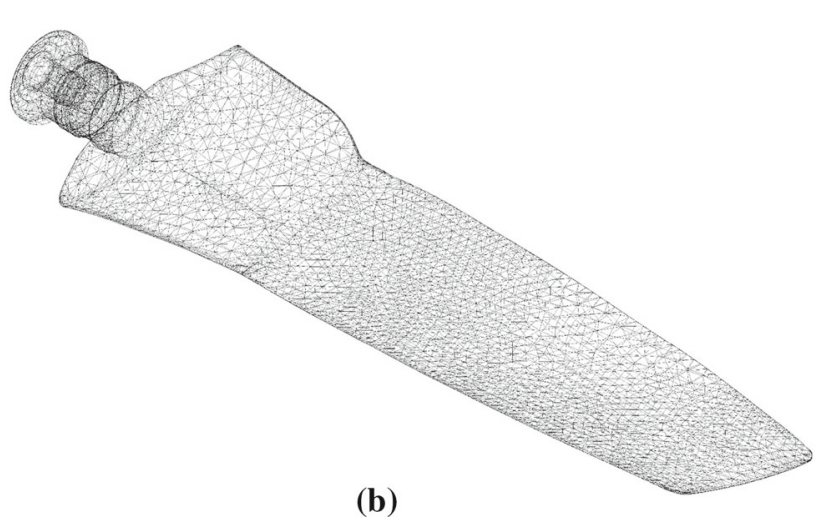

Fig. 1 54H60 propeller blade. a Configuration, b Surface discretization 
the estimation of the frequency response, to validate both modules independently.

First, the pre-stressed state under $\Omega_{\text {rot }}$ of 1,020 RPM will be obtained by the nonlinear static FOM solver, where $\Omega_{\text {rot }}$ is the rotating speed. It takes seven Newton-Raphson iterations for the force convergence, such that $\left\|\mathbf{f}^{\text {ext }}-\mathbf{f}^{\text {int }}\right\|_{2} /\left\|\mathbf{f}^{\text {ext }}\right\|_{2}<$ $10^{-6}$. As shown in Fig. 2, the present FOM module generates similar results compared with those from ANSYS; and the normalized root mean square error (NRMSE) in entire FE nodes are found to be $0.11 \%$ and $0.11 \%$ for the displacement and von-Mises stress, respectively. Herein, NRMSE is RMSE normalized by RMS of the reference value.

The linear frequency response is obtained under an oscillatory transverse tip force of amplitude $1,000 \mathrm{~N}$. The frequency range extends from 5 to $300 \mathrm{~Hz}$ with an interval of $5 \mathrm{~Hz}$. Figure 3 a shows the maximum transverse displacement for each frequency and is completely identical for te present FOM and

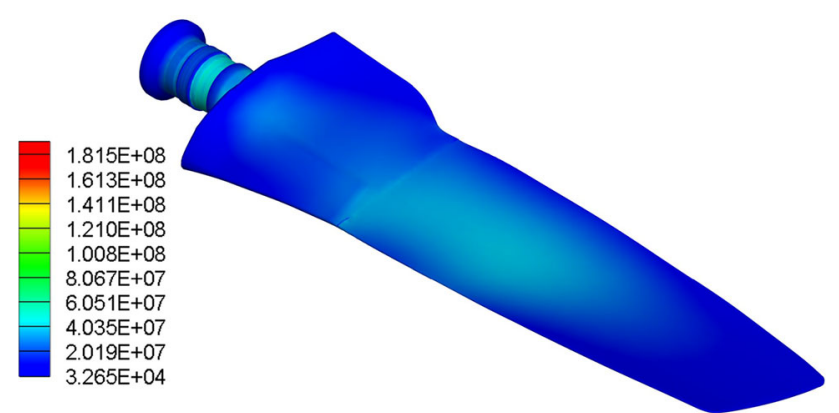

(a)

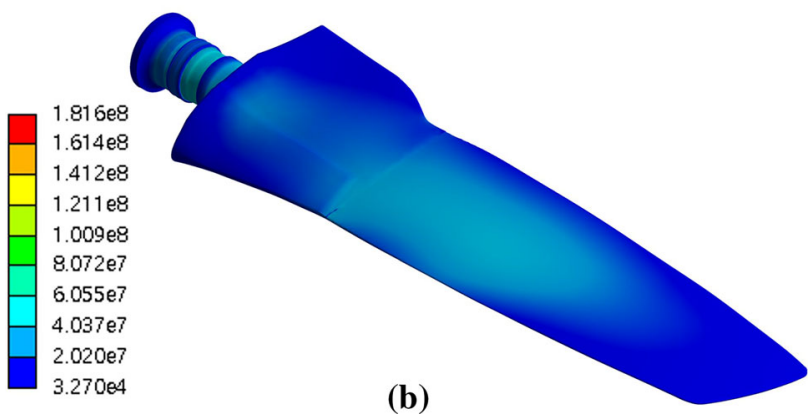

Fig. 2 FOM validation for the nonlinear static analysis: von-Mises stress distribution. All units in Pascal. a Present, b ANSYS

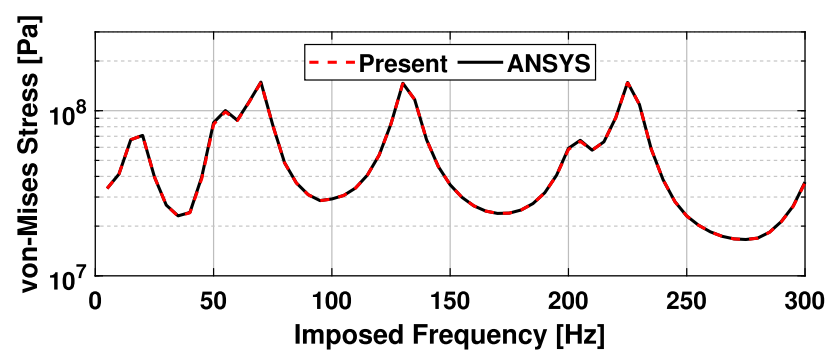

Fig. 3 FOM validation for the frequency response analysis: maximum transverse displacement amplitude



Fig. 4 FOM validation for the frequency response analysis: maximum von-Mises stress by an alternating stress

ANSYS. The maximum von-Mises stress, which is crucial for the investigation of HCF, is derived from the alternating stress component as shown in Fig. 4. NRMSE over the frequency band is $0.72 \%$, which is due to the difference of the stress recovery method for the harmonic values. Based on the validation, it is concluded that the present FOM solver will be reliable for the forced vibration analysis of the rotating component.

\section{Projection-based model order reduction}

Projection-based MOR is an effective technique to reduce the dimension of the aforementioned FOM for the forced vibration analysis of the rotating component. The method may be categorized by the projection matrices (e.g. eigenmode and POD mode matrix). Furthermore, the hyper-reduction method will further decrease the computational cost of ROM assemblage (e.g. FOM assemblage and Galerkin projection) by the sparse sampling method. In this section, MSM, POD, and their hybrid ROM approaches will be discussed. Then, application of the energy-conserving sampling and weighting (ECSW) scheme, which was the hyper-reduction method recommended by Ref. [14], will be proposed for the computation of the forced vibration analysis.

\subsection{Mode superposition method (MSM)}

MSM is one of the widely used methods to reduce the dimension in the frequency response analysis. Given the prestressed stiffness matrix $\mathbf{K}$ from the nonlinear static analysis, the equation of the modal analysis, for the eigenmode extraction, are expressed in Eq. 8.

$$
\begin{gathered}
\left(\mathbf{K}\left(\mathbf{u}_{m}(\mu) ; \mu\right)-\left(\omega_{i}(\mu)\right)^{2} \mathbf{M}(\mu)\right) \boldsymbol{\phi}_{i}(\mu)=\mathbf{0} \\
i \in\left\{1,2, \ldots, n_{\phi}\right\}
\end{gathered}
$$

Herein, a non-trivial solution $\omega_{i}$ and $\boldsymbol{\phi}_{i} \in \mathbb{R}^{N}$ are the $i$ th natural circular frequency and eigenmode, respectively. $n_{\phi}$ indicates the number of the natural modes obtained. Then, the projection matrix of MSM will become $\boldsymbol{\Phi}=$ $\left[\boldsymbol{\phi}_{1}, \boldsymbol{\phi}_{2}, \ldots, \boldsymbol{\phi}_{n_{\phi}}\right] \in \mathbb{R}^{N \times n_{\phi}}$. 
Since $\boldsymbol{\Phi}^{T} \mathbf{M} \boldsymbol{\Phi}=\mathbf{I}$ and $\boldsymbol{\Phi}^{T} \mathbf{K} \boldsymbol{\Phi}=\operatorname{diag}\left\{\omega_{1}^{2}, \omega_{2}^{2}, \ldots, \omega_{n_{\phi}}^{2}\right\}$, where $\mathbf{I} \in \mathbb{R}^{n_{\phi} \times n_{\phi}}$ is the identity matrix, the ROM of the frequency response analysis (Eq. 7) may be expressed as follows:

$$
\begin{aligned}
& \left(-\Omega^{2} \mathbf{I}+j \Omega \boldsymbol{\Phi}^{T}(\mu) \mathbf{C}(\mu) \boldsymbol{\Phi}(\mu)+\boldsymbol{\Lambda}^{2}(\mu)\right) \\
& \quad \times \mathbf{y}_{c}(\mu ; \Omega)=\boldsymbol{\Phi}^{T}(\mu) \mathbf{f}_{c}(\mu) \\
& \mathbf{u}_{c}(\mu ; \Omega)=\boldsymbol{\Phi}(\mu) \mathbf{y}_{c}(\mu ; \Omega)
\end{aligned}
$$

where $\boldsymbol{\Lambda}^{2}=\operatorname{diag}\left\{\omega_{1}^{2}, \omega_{2}^{2}, \ldots, \omega_{n_{\phi}}^{2}\right\}$ is the diagonal eigenvalue matrix; and $\mathbf{y}_{c} \in \mathbb{C}^{n_{\phi}}$ is the reduced complex displacement vector in the modal coordinates. It is found that the final solution $\mathbf{u}_{c}$ is approximated as a linear combination of the eigenmodes, in which the coefficients are the reduced solution $\mathbf{y}_{c}$.

MSM effectively reduces the dimension in the frequency response analysis from $N$ to $n_{\phi}$. However, the computational cost of the nonlinear static analysis (Eq. 2) remains the same, and a modal analysis (Eq. 8) will be required. Moreover, the estimation of the projection matrix $\boldsymbol{\Phi}$, which requires both nonlinear static and modal analysis in FOM, should be performed for each parameter $(\mu)$ variation.

\subsection{Proper orthogonal decomposition (POD)}

In contrast, the computation of POD modes [8] is separated from the FOM of nonlinear static and modal analysis, and it will be conducted in the so-called off-line stage. Then, an online stage will follow, in which ROM will be defined by Galerkin projection of FOM to POD coordinates.

To be more specific, the off-line stage starts with the collection of the sample result that represents the dynamic behavior of FOM. In this paper, the result will be collected by the nonlinear time-transient analysis module, of which the governing equation and numerical algorithm are found in detail in Ref. [7]. Then the snapshot matrix $\mathbf{W} \in \mathbb{R}^{N \times N_{s}}$ will be constructed by the time- and parameter-varying displacement vectors as follows:

$\mathbf{W}=\left[\mathbf{u}\left(t_{1} ; \mu_{1}\right), \ldots, \mathbf{u}\left(t_{N_{s}} ; \mu_{N_{s}}\right)\right]$

where $t$ and $N_{s}$ are time and the number of the snapshot samples. Then, POD modes will be obtained from the orthogonal basis vectors of the compact singular value decomposition, as Eq. 11.

$\mathbf{W}=\mathbf{U S V}$
$\mathbf{\Psi}=\left[\boldsymbol{\psi}_{1}, \boldsymbol{\psi}_{2}, \ldots, \boldsymbol{\psi}_{n_{\psi}}\right]$

$\mathbf{U} \in \mathbb{R}^{N \times N_{s}}$ and $\mathbf{V} \in \mathbb{R}^{N_{s} \times N_{s}}$ are the left and right singular vector matrices; $\mathbf{S} \in \mathbb{R}^{N_{s} \times N_{s}}$ is the diagonal singular value matrix; and $n_{\psi}$ is the number of POD modes for utilization.
$\Psi \in \mathbb{R}^{N \times n_{\psi}}$ is the POD mode matrix, which comprise the first $n_{\psi}$ column vectors of $\mathbf{U}$.

In the online stage, the governing equations of FOM will be transformed to POD mode coordinates by Galerkin projection (e.g. $\boldsymbol{\Psi}^{T} \mathbf{K} \boldsymbol{\Psi}$ and $\boldsymbol{\Psi}^{T} \mathbf{u}$ ). Then, POD-based ROM of the forced vibration analysis will be expressed as Eqs. 12 and 13 for the nonlinear static (Eq. 2) and the frequency response analysis (Eq. 7), respectively [6].

$$
\begin{gathered}
\boldsymbol{\Psi}^{T} \mathbf{K}\left(\mathbf{u}_{k}(\mu) ; \mu\right) \boldsymbol{\Psi} \Delta \mathbf{u}_{k}^{r}(\mu)=\boldsymbol{\Psi}^{T}\left(\mathbf{f}_{0}^{\text {ext }}(\mu)\right. \\
\left.\quad+\mathbf{K}^{\mathrm{sp}}(\mu) \mathbf{u}_{k}(\mu)-\mathbf{f}^{\text {int }}\left(\mathbf{u}_{k}(\mu) ; \mu\right)\right) \\
\Delta \mathbf{u}_{k}(\mu)=\boldsymbol{\Psi} \Delta \mathbf{u}_{k}^{r}(\mu)
\end{gathered}
$$

$$
\begin{aligned}
& \left(-\Omega^{2} \boldsymbol{\Psi}^{T} \mathbf{M}(\mu) \boldsymbol{\Psi}+j \Omega \boldsymbol{\Psi}^{T} \mathbf{C}(\mu) \boldsymbol{\Psi}\right. \\
& \left.\quad+\boldsymbol{\Psi}^{T} \mathbf{K}\left(\mathbf{u}_{m}(\mu) ; \mu\right) \boldsymbol{\Psi}\right) \mathbf{u}_{c}^{r}(\mu ; \Omega)=\boldsymbol{\Psi}^{T} \mathbf{f}_{c}(\mu) \\
& \mathbf{u}_{c}(\mu ; \Omega)=\boldsymbol{\Psi} \mathbf{u}_{c}^{r}(\mu ; \Omega)
\end{aligned}
$$

Herein, $\Delta \mathbf{u}_{k}^{r} \in \mathbb{R}^{n_{\psi}}$ and $\mathbf{u}_{c}^{r} \in \mathbb{C}^{n_{\psi}}$ denote the displacement increment and the complex displacement vectors in POD mode coordinates.

Also, in the case of $n_{\psi} \geq n_{\phi}$, the governing equations of the modal analysis (Eq. 8) will be written in the form of POD-based ROM as follows [5]:

$$
\begin{gathered}
\left(\boldsymbol{\Psi}^{T} \mathbf{K}\left(\mathbf{u}_{m}(\mu) ; \mu\right) \Psi\right. \\
\left.-\left(\omega_{i}(\mu)\right)^{2} \boldsymbol{\Psi}^{T} \mathbf{M}(\mu) \Psi\right) \boldsymbol{\phi}_{i}^{r}(\mu)=\mathbf{0} \\
\quad i \in\left\{1,2, \ldots, n_{\phi}\right\} \\
\boldsymbol{\phi}_{i}(\mu)=\boldsymbol{\Psi}^{T} \boldsymbol{\phi}_{i}^{r}(\mu) \quad i \in\left\{1,2, \ldots, n_{\phi}\right\}
\end{gathered}
$$

where the non-trivial solution $\boldsymbol{\phi}_{i}^{r} \in \mathbb{R}^{n_{\psi}}$ is the $i$-th eigenmode in POD mode coordinates.

Unlike the eigenmode matrix $\boldsymbol{\Phi}$ of MSM, POD mode matrix $\boldsymbol{\Psi}$ will be independent of the parameter $\mu$ during the online stage. In this respect, POD is an effective method for the multi-query simulation, in which a number of the simulation parameters will be investigated. Also, POD will be applicable not only to the governing equations of the forced vibration analysis (Eqs. 12 and 13), but also to those of the modal analysis (Eq. 14). It will initiate hybrid approaches of POD and MSM, in which the natural frequencies and eigenmodes will be obtained by POD-based ROM (Eqs. 12 and 14), and the frequency responses by MSM (Eq. 9).

The computational cost for solving $n_{\phi}$ or $n_{\psi}$ equations will be negligible compared to that for $N$ equations. However, the computational bottleneck will occur by the construction of ROM equations. This will include assemblage of FOM 
matrices and vectors (e.g. computation of $\mathbf{K}$ and $\mathbf{f}^{\text {int }}$ ) and Galerkin projection (e.g. $\boldsymbol{\Psi}^{T} \mathbf{K} \boldsymbol{\Psi}$ and $\boldsymbol{\Psi}^{T} \mathbf{f}^{\text {int }}$ ). The bottleneck will become significant in the nonlinear analysis (Eq. 12), in which ROM construction will be required in each NewtonRaphson iteration.

\subsection{Energy-conserving sampling and weighting method (ECSW)}

To minimize the cost for ROM assemblage of the nonlinear analysis, the hyper-reduction scheme will employ the sparse sampling method. ECSW [14] defines a reduced set of the elements with the corresponding weightings by an energy-conserving approach which is based on the principle of virtual work.

Therefore, additional effort of the coarse set definition will be required in the off-line stage. First, the reduced training vector will be obtained from the collected result in Eq. 10, using the least square as follows:

$$
\begin{aligned}
\mathbf{q}_{i} & =\left(\boldsymbol{\Psi}^{T} \boldsymbol{\Psi}\right)^{-1} \boldsymbol{\Psi}^{T} \mathbf{u}\left(t_{i} ; \mu_{i}\right) \\
i & \in\left\{1,2, \ldots, N_{s}\right\}
\end{aligned}
$$

where $\mathbf{q}_{i} \in \mathbb{R}^{n_{\psi}}$ is the $i$-th reduced training vector. Then, the elemental contribution $\mathbf{g}_{i e} \in \mathbb{R}^{n_{\phi}}$ of the reduced nonlinear term will be defined with their element-wise summation $\mathbf{b}_{i} \in$ $\mathbb{R}^{n_{\phi}}$ as follows:

$$
\begin{aligned}
& \mathbf{g}_{i e}=\Psi^{T} \mathbf{L}_{e}^{T} \mathbf{f}_{e}^{\mathrm{int}}\left(\mathbf{L}_{e} \boldsymbol{\Psi} \mathbf{q}_{i}(\mu) ; \mu\right) \\
& i \in\left\{1,2, \ldots, N_{s}\right\}, \quad e \in \mathbf{E} \\
& \mathbf{b}_{i}=\sum_{e \in \mathbf{E}} \mathbf{g}_{i e} \quad i \in\left\{1,2, \ldots, N_{s}\right\}
\end{aligned}
$$

Moreover, a block matrix $\mathbf{G} \in \mathbb{R}^{n_{\psi} N_{s} \times N_{e}}$ and a vector $\mathbf{b} \in$ $\mathbb{R}^{n_{\psi} N_{s}}$ will be assembled as follows:

$$
\begin{aligned}
& \mathbf{G}=\left[\begin{array}{ccc}
\mathbf{g}_{11} & \ldots & \mathbf{g}_{1 N_{e}} \\
\vdots & \ddots & \vdots \\
\mathbf{g}_{N_{s} 1} & \ldots & \mathbf{g}_{N_{s} N_{e}}
\end{array}\right] \\
& \mathbf{b}=\left\{\begin{array}{c}
\mathbf{b}_{1} \\
\vdots \\
\mathbf{b}_{N_{s}}
\end{array}\right\}
\end{aligned}
$$

Based on the matrix $\mathbf{G}$ and vector $\mathbf{b}$, the standard nonnegative least square (NNLS) equation will be defined as Eq. 18.

$$
\boldsymbol{\xi}=\arg \min _{\boldsymbol{\alpha} \geq \mathbf{0}}\|\mathbf{G} \boldsymbol{\alpha}-\mathbf{b}\|_{2}^{2}
$$

Herein, $\boldsymbol{\alpha} \in \mathbb{R}^{N_{e}}$ and $\boldsymbol{\xi}=\left[\xi_{1}, \xi_{2}, \ldots, \xi_{N_{e}}\right]^{T} \in \mathbb{R}^{N_{e}}$ are the coefficient vector and the set of element weightings associated with $N_{e}$ elements. An iterative solution algorithm of the standard NNLS was proposed by Lawson and Hanson [20]. Moreover, the variant of the algorithm, called the sparse NNLS, was suggested by Farhat et al. [14] by adding the iteration termination criterion with a positive tolerance $\epsilon$ as follows:

$\|\mathbf{G} \boldsymbol{\alpha}-\mathbf{b}\|_{2} \leq \epsilon\|\mathbf{b}\|_{2}$

Through the additional off-line stage of Eqs. 15-19, the reduced set of elements will be defined as $\tilde{\mathbf{E}}=\left\{\tilde{e}: \xi_{\tilde{e}}>\right.$ $0\} \subset \mathbf{E}$.

In the online stage, ROM assemblage for the nonlinear static analysis (Eq. 12) will be expressed as follows:

$$
\begin{aligned}
& \boldsymbol{\Psi}^{T} \tilde{\mathbf{K}}\left(\mathbf{u}_{k}(\mu) ; \mu\right) \boldsymbol{\Psi} \Delta \mathbf{u}_{k}^{r}(\mu)=\boldsymbol{\Psi}^{T}\left(\mathbf{f}_{0}^{\mathrm{ext}}(\mu)\right. \\
& \left.+\tilde{\mathbf{K}}^{\mathrm{sp}}(\mu) \mathbf{u}_{k}(\mu)-\tilde{\mathbf{f}}^{\mathrm{int}}\left(\mathbf{u}_{k}(\mu) ; \mu\right)\right) \\
& \Delta \mathbf{u}_{k}(\mu)=\boldsymbol{\Psi} \Delta \mathbf{u}_{k}^{r}(\mu)
\end{aligned}
$$

where the element-wise assemblage of $\tilde{\mathbf{K}} \in \mathbb{R}^{N \times N}, \tilde{\mathbf{K}}^{\text {sp }} \in$ $\mathbb{R}^{N \times N}, \tilde{\mathbf{f}} \tilde{\mathbf{f}}^{\text {int }} \in \mathbb{R}^{N}$ is performed in the reduced element set $\tilde{\mathbf{E}}$ as Eq. 21.

$\tilde{\mathbf{K}}\left(\mathbf{u}_{k}(\mu) ; \mu\right)=\sum_{\tilde{e} \in \tilde{\mathbf{E}}} \xi_{\tilde{e}} \mathbf{L}_{\tilde{e}}^{T} \mathbf{K}_{\tilde{e}}\left(\mathbf{L}_{\tilde{e}} \mathbf{u}_{k}(\mu) ; \mu\right) \mathbf{L}_{\tilde{e}}$

$\tilde{\mathbf{K}}^{\mathrm{sp}}(\mu)=\sum_{\tilde{e} \in \tilde{\mathbf{E}}} \xi_{\tilde{e}} \mathbf{L}_{\tilde{e}}^{T} \mathbf{K}_{\tilde{e}}^{\mathrm{sp}}(\mu) \mathbf{L}_{\tilde{e}}$

$\tilde{\mathbf{f}}^{\mathrm{int}}\left(\mathbf{u}_{k}(\mu) ; \mu\right)=\sum_{\tilde{e} \in \tilde{\mathbf{E}}} \xi_{\tilde{e}} \mathbf{L}_{\tilde{e}}^{T} \mathbf{f}_{\tilde{e}}^{\mathrm{int}}\left(\mathbf{L}_{\tilde{e}} \mathbf{u}_{k}(\mu) ; \mu\right)$

Equation 21 shows that the number of element-wise summations for the assemblage of FOM matrices and vectors will be drastically decreased. Also, considering that FOM matrices are usually allocated in a sparse storage format, non-zero entries of the matrix will be significantly reduced, leading to the computational cost reductions for the matrix-matrix or matrix-vector multiplication in Eq. 20.

\section{Application to the rotating component}

In this section, a comparison of the computational performance will be conducted among the variety of MOR frameworks when applied to rotating component forced vibration parametric analysis. MOR application is depicted in Fig. 5 and listed as follows: 
Fig. 5 Present MOR framework
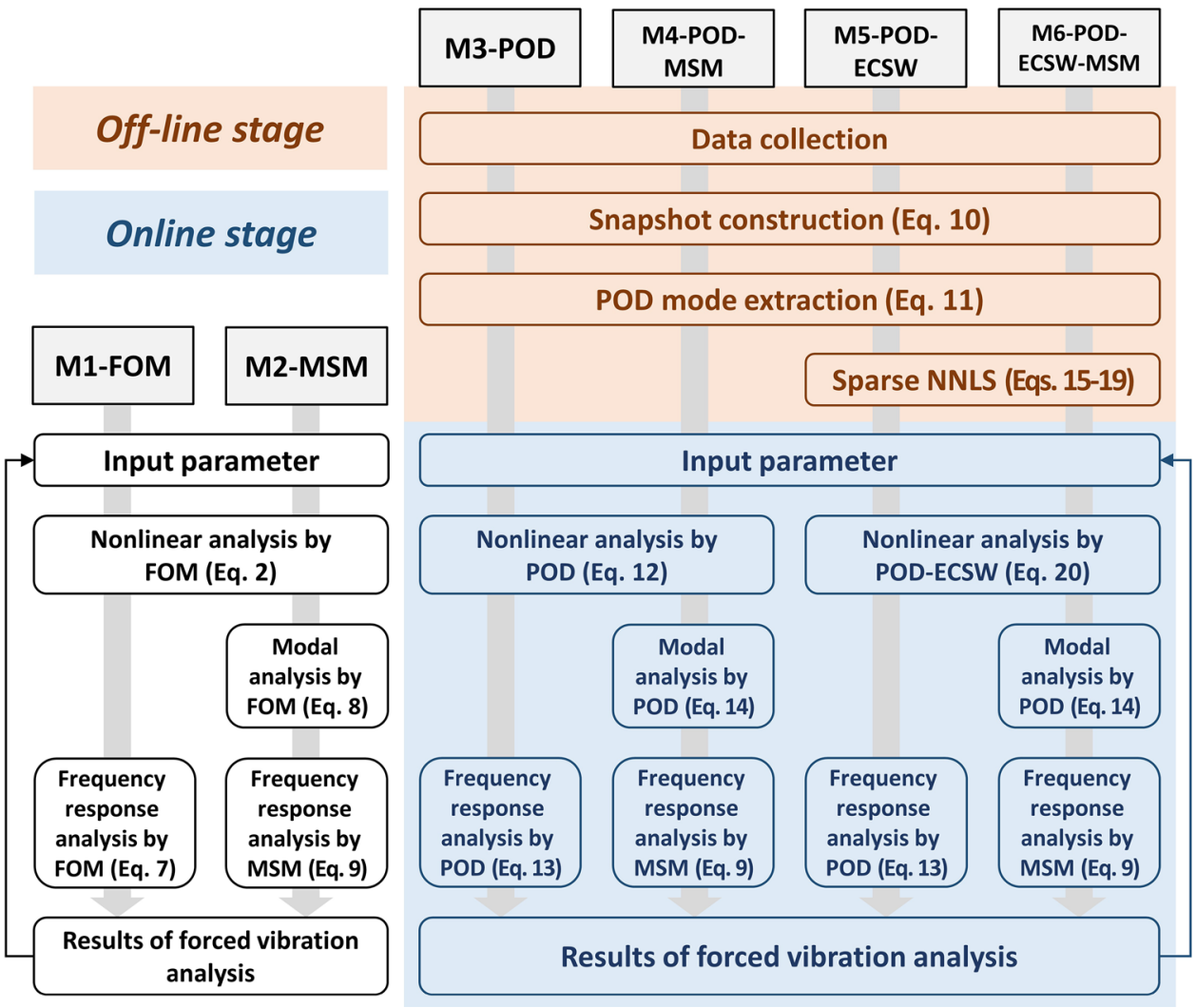

- M1-FOM: The forced vibration analysis will be conducted solely by FOM. The pre-stressed stiffness matrix $\mathbf{K}$ will be obtained by the nonlinear analysis (Eq. 2). Then, the frequency response $\mathbf{u}_{c}$ will be obtained by the linear harmonic analysis (Eq. 7).

- M2-MSM: This will be the same as M1-FOM for $\mathbf{K}$ by the nonlinear analysis (Eq. 2). Then, the eigenmodes $\boldsymbol{\Phi}$ and eigenvalues $\boldsymbol{\Lambda}^{2}$ will be obtained by the modal analysis of FOM (Eq. 8). Next, the frequency response $\mathbf{u}_{c}$ will be obtained in the eigenmode coordinates (Eq. 9).

- M3-POD: POD modes $\boldsymbol{\Psi}$ will be extracted from the offline stage (Eqs. 10-11). Then, $\mathbf{K}$ and $\mathbf{u}_{c}$ will be estimated by the nonlinear static (Eq. 12) and linear frequency response analysis (Eq. 13) sequentially in POD mode coordinates.

- M4-POD-MSM: This will be the same as M3-POD for $\Psi$ and $\mathbf{K}$ by the off-line stage (Eqs. 10-11) and the nonlinear analysis (Eq. 12), respectively. Then, the modal analysis for $\boldsymbol{\Phi}$ and $\boldsymbol{\Lambda}^{2}$ will be conducted by POD-based ROM (Eq. 14). The remaining procedure for $\mathbf{u}_{c}$ (Eq. 9) will be the same as M2-MSM.

- M5-POD-ECSW: The off-line stage for $\boldsymbol{\Psi}$ (Eqs. 10-11) will be extended by the sparse NNLS (Eqs. 15-19) to obtain the weighting vector $\boldsymbol{\xi}$ and the set of the reduced elements $\tilde{\mathbf{E}}$. Then, the nonlinear static analysis will be performed by the hyper-reduction method (Eq. 20) using the stiffness matrix $\tilde{\mathbf{K}}$ obtained by the reduced assem-

blage. The remaining procedure for $\mathbf{u}_{c}$ (Eq. 13) will be the same as M3-POD, except that the matrix $\mathbf{K}$ will be replaced by $\tilde{\mathbf{K}}$.

- M6-POD-ECSW-MSM: This will be the same as M5POD-ECSW for $\boldsymbol{\Psi}$ (Eqs. 10-11), $\boldsymbol{\xi}, \tilde{\mathbf{E}}$ (Eqs. 15-19) and $\tilde{\mathbf{K}}$ (Eq. 20). The remaining procedures for $\boldsymbol{\Phi}, \boldsymbol{\Lambda}^{2}$ (Eq. 14) and $\mathbf{u}_{c}$ (Eq. 9) will be the same as M4-POD-MSM, except that the matrix $\mathbf{K}$ will be replaced by $\tilde{\mathbf{K}}$.

The first application is the previously shown $54 \mathrm{H} 60$ propeller blade, which will be discretized into hundreds of thousands of DOFs. The blade has a slender configuration that will induce a high degree of geometrical nonlinearities. The second object is the first stage turbine blade in the GT11N, of which its discretization has millions of DOFs due to its complicated design incurred by inner hole features.

The specification for the present computational resource and libraries will be as follows: A single core Intel i910900KF 3.70GHz CPU will be used for the entire computation. For the computation of the dense matrices (Eqs. 9, 1114, 18-20), Intel MKL LAPACK [17] will be employed. In the case of the large-size sparse matrices (Eqs. 2, 7-8, and FOM analysis for the result collection), Intel MKL PARDISO [17] will be used. ARPACK [21] will be additionally adopted for the eigensolution in Eq. 8 . 
To measure the accuracy of the present MOR, two indices will be defined as follows:

$$
\begin{aligned}
& E I_{u}(\mu) \\
& =\frac{\sqrt{\sum_{\Omega \in \mathbf{F}}\left\|\mathbf{u}_{c}^{\mathrm{FOM}}(\mu ; \Omega)-\mathbf{u}_{c}^{\mathrm{ROM}}(\mu ; \Omega)\right\|_{2}^{2}}}{\sqrt{\sum_{\Omega \in \mathbf{F}}\left\|\mathbf{u}_{c}^{\mathrm{FOM}}(\mu ; \Omega)\right\|_{2}^{2}}} \times 100 \\
& E I_{\sigma}(\mu) \\
& =\frac{\sqrt{\sum_{\Omega \in \mathbf{F}}\left\|\boldsymbol{\sigma}_{v}^{\mathrm{FOM}}(\mu ; \Omega)-\boldsymbol{\sigma}_{v}^{\mathrm{ROM}}(\mu ; \Omega)\right\|_{2}^{2}}}{\sqrt{\sum_{\Omega \in \mathbf{F}}\left\|\boldsymbol{\sigma}_{v}^{\mathrm{FOM}}(\mu ; \Omega)\right\|_{2}^{2}}} \times 100
\end{aligned}
$$

where the superscripts FOM and ROM denote the quantities obtained by FOM (i.e. M1-FOM) and ROM. F = $\left\{\Omega_{1}, \Omega_{2}, \ldots, \Omega_{N_{f}}\right\}$ is the set of the imposed frequencies, where $N_{f}$ indicates the number of the frequencies. $\sigma_{v} \in \mathbb{R}^{N_{n}}$ contains the maximum von-Mises stress in each computational node which is derived from the alternating stress components. $N_{n}=N / 3$ is the total number of FE nodes.

The speedup performance $S$ and the effective speedup one $S_{\text {eff }}$ will be defined as follows:

$$
\begin{aligned}
& S=\frac{T_{\mathrm{FOM}}}{T_{\mathrm{ROM}}} \\
& S_{\text {eff }}=\frac{n_{\text {sim }} T_{\mathrm{FOM}}}{T_{\text {off }}+n_{\text {sim }} T_{\mathrm{ROM}}}
\end{aligned}
$$

where $T_{\mathrm{FOM}}, T_{\mathrm{ROM}}$ and $T_{\text {off }}$ are the computational time spent for FOM, ROM, and the off-line stage $\left(T_{\text {off }}=0\right.$ for M2MSM), respectively. $n_{\text {sim }}$ is the number of the simulations of the parametric analysis.

\section{1 $54 \mathrm{H} 60$ propeller blade}

The 54H60 propeller blade is analyzed first. The parameter $\mu=\Omega_{\text {rot }}$ varies from 510 to 1,530 RPM at an interval of 102 RPM. The discretization and the harmonic excitation of the tip are the same as in Sect. 2.3. The number of eigenmodes is selected to be 13 , where the corresponding maximum natural frequency (from 501 to $521 \mathrm{~Hz}$ by Eq. 8) sufficiently covers the frequency band of the imposed excitation. POD-applied MOR is examined by the various number of POD bases, from 20 to 50 at an interval of 10 .

Regarding the off-line stage, the snapshot matrix $\mathbf{W}$ of 50 column vectors will be constructed $\left(N_{s}=50\right)$. The nonlinear time-transient analysis is conducted to obtain the timevarying displacement vectors of $\mathbf{W}$, in which the sinusoidal forces and angular velocities are imposed. To be more specific, tip force of amplitude $1,000 \mathrm{~N}$ is imposed along the longitudinal axis, and the angular velocity of amplitude 1,020 RPM is imposed along the transverse and rotating axes for each. The sinusoidal function oscillates during 5 periods with



Fig. 6 Time-varying tip displacement of the $54 \mathrm{H} 60$ propeller blade obtained from the snapshot matrix

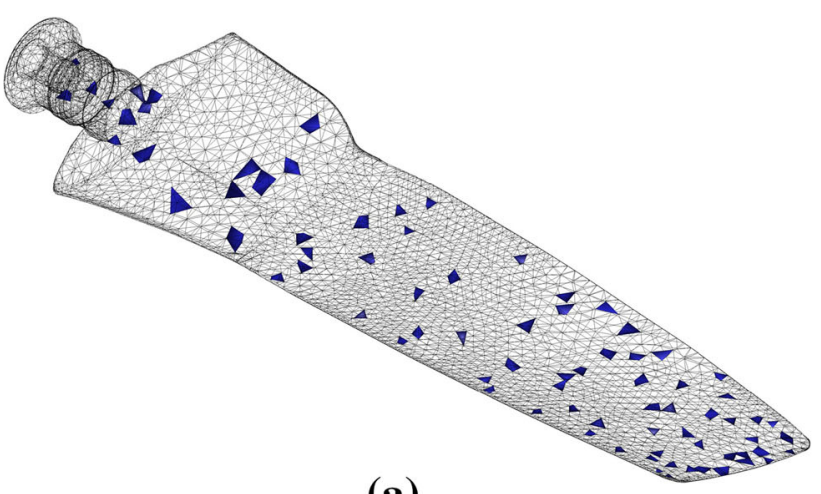

(a)

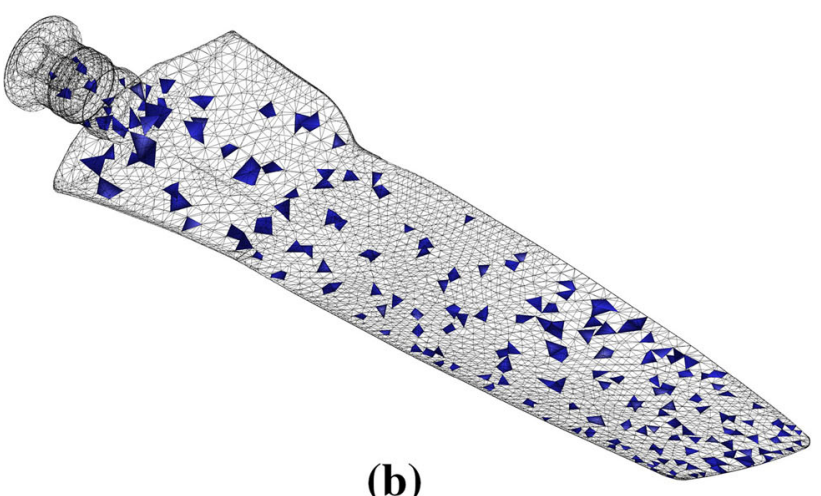

Fig. 7 Sampled mesh for the 54H60 propeller blade obtained by the present sparse NNLS. a $n_{\psi}=20\left(N_{\tilde{e}}=86\right), \mathbf{b} n_{\psi}=50\left(N_{\tilde{e}}=212\right)$

the frequency of $17 \mathrm{~Hz}$. Figure 6 shows the time-varying response obtained for the nonlinear time-transient analysis.

Except for the sparse NNLS, it takes 983.9 seconds for the off-line stage, which is $T_{\text {off }}$ of POD-applied MOR without the hyper-reduction method (M3-POD and M4-POD-MSM). To apply ECSW (M5-POD-ECSW and M6-POD-ECSWMSM), the computational time of the sparse NNLS ( $T_{\mathrm{NNLS}}$ ) will be added to the off-line cost. The sets of the reduced ele- 
Table 1 Accuracy and speedup performance of MOR for the 54H60 propeller blade ( $\mu=1,020 \mathrm{RPM})$

\begin{tabular}{llll}
\hline MOR framework & $E I_{u}(\%)$ & $E I_{\sigma}(\%)$ & $S$ \\
\hline M2-MSM & 0.97 & 6.36 & 9.9 \\
M3-POD & $20.80 / 7.71 / 2.20 / 0.81$ & $53.40 / 19.49 / 6.64 / 3.89$ & $30.2 / 27.8 / 25.7 / 23.7$ \\
M4-POD-MSM & $20.80 / 7.73 / 2.20 / 0.97$ & $53.43 / 19.80 / 7.10 / 5.42$ & $32.3 / 29.9 / 27.5 / 25.5$ \\
M5-POD-ECSW & $21.33 / 6.94 / 3.05 / 1.10$ & $53.13 / 17.00 / 8.00 / 4.05$ & $229.0 / 183.7 / 158.0 / 128.5$ \\
M6-POD-ECSW-MSM & $21.33 / 6.96 / 3.06 / 1.22$ & $53.15 / 17.36 / 8.39 / 5.47$ & $232.4 / 202.6 / 171.8 / 142.4$ \\
\hline
\end{tabular}

The values in the multi-entry cells correspond to $n_{\psi}$ of $20,30,40$ and 50

ments $\tilde{\mathbf{E}}$ are obtained by the positive tolerance $\epsilon=0.001$ of Eq. 19. Herein, the number of reduced elements $N_{\tilde{e}}$ increases in terms of the number of POD bases employed as shown in Fig. 7. The computational time for the sparse NNLS is 12.3, 15.8, 22.0, and 37.5 seconds for each $n_{\psi}$.

The accuracy $\left(E I_{u}\right.$ and $\left.E I_{\sigma}\right)$ and speedup $(S)$ performance of MOR is investigated. The case for $\mu=1,020 \mathrm{RPM}$ is summarized in Table. 1. When enough POD bases are provided, a significant improvement in speedup performance will be obtained by the hyper-reduction methods. Despite a small accuracy loss, this method results in a dramatic cost reduction for the nonlinear static analysis. The computational time for the nonlinear static analysis is 16.9, 8.5, and 0.6 seconds for Eqs. 2, 12 and 20, respectively, for $n_{\psi}=50$.

To examine the robustness of the present MOR, indices $E I_{u}, E I_{\sigma}$, and $S$ are compared in terms of the parameter $\mu$ as shown in Figs. 8, 9, 10. An increase of the displacement and stress discrepancy is observed for large $\mu$, which is due to the high nonlinearity induced by the large centrifugal force. Nevertheless, the discrepancy remains within the admissible boundary for values of $\mu\left(E I_{u}<1.67 \%\right.$ and $\left.E I_{\sigma}<6.99 \%\right)$. To be specific, the maximum transverse displacement is indicated for each frequency location as shown in Figs. 11, 12, 13. As the parameter $\mu$ increases, the peak locations appear in the higher frequency locations, which implies that the stiffness increases in terms of the rotating speed. To be more specific, the significant nonlinear stress-stiffening effect occurs,

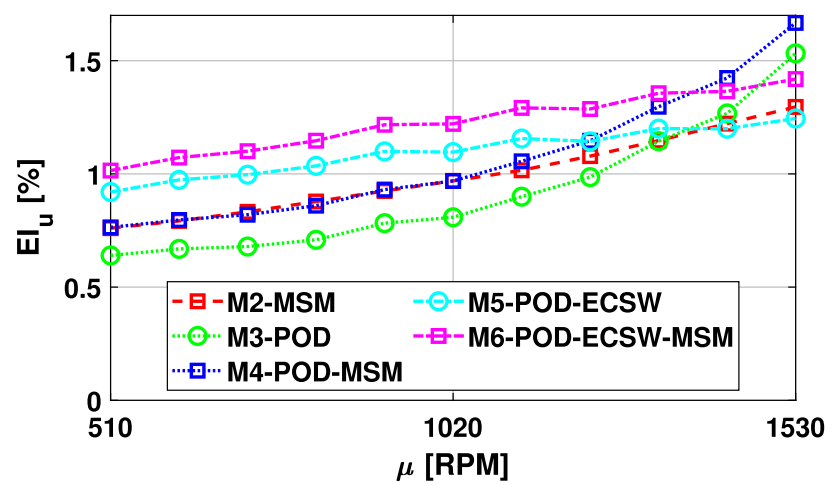

Fig. 8 Accuracy performance of the present MOR for the 54H60 propeller blade in terms of $\mu\left(n_{\psi}=50\right): E I_{u}$

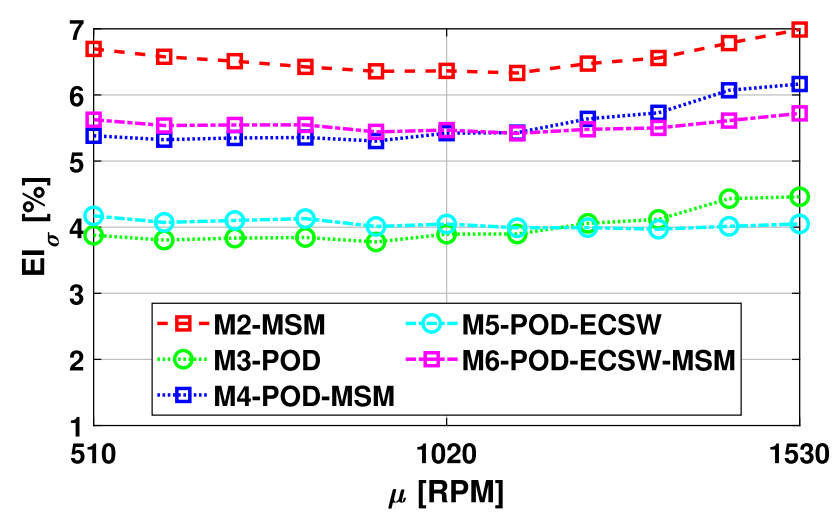

Fig. 9 Accuracy performance of the present MOR for the 54H60 propeller blade in terms of $\mu\left(n_{\psi}=50\right): E I_{\sigma}$

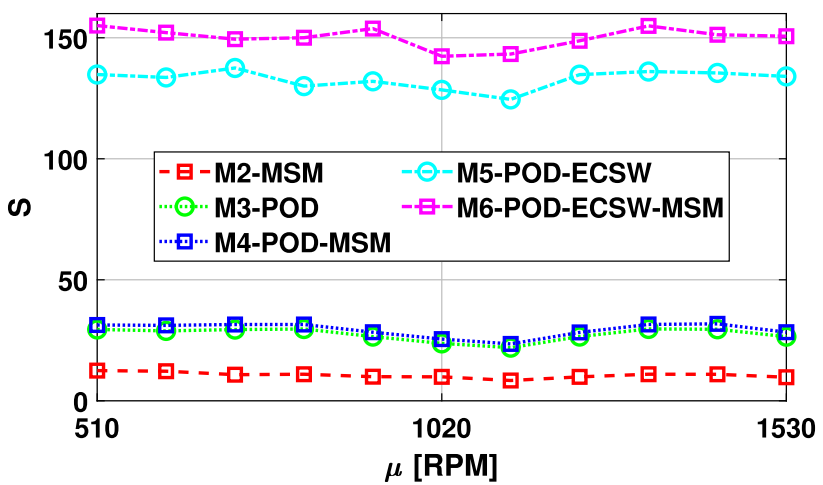

Fig. 10 Speed performance of the present MOR for the $54 \mathrm{H} 60$ propeller blade in terms of $\mu\left(n_{\psi}=50\right): S$



Fig. 11 Maximum transverse displacement amplitude in the 54H60 propeller blade $\left(n_{\psi}=50\right): \mu=510 \mathrm{RPM}$ 




Fig. 12 Maximum transverse displacement amplitude in the 54H60 propeller blade $\left(n_{\psi}=50\right): \mu=1,020 \mathrm{RPM}$



Fig. 13 Maximum transverse displacement amplitude in the 54H60 propeller blade $\left(n_{\psi}=50\right): \mu=1,530 \mathrm{RPM}$

which further exceeds the spin-softening effect. Such trends will not be observed without the pre-computation of the nonlinear static analysis. All MOR frameworks follow similar trends, however, slight deviations occur in the higher frequency region for large $\mu$. Still, accurate results are obtained at the peaks as shown in Fig. 14, in which the maximum vonMises stress by an alternating stress is obtained in each FE node at the last peak frequency of $250 \mathrm{~Hz}(\mu=1,530 \mathrm{RPM})$.

The final investigation is for the effective speedup to measure the substantial acceleration performance by including the computational cost of the off-line stage. To obtain $S_{\text {eff }}$, the computational time of FOM and ROM is averaged among the parameter $\mu$. Figure 15 shows the effective speedup with respect to the number of simulations. Since there is no offline stage for MOR with only MSM (M2-MSM), its effective

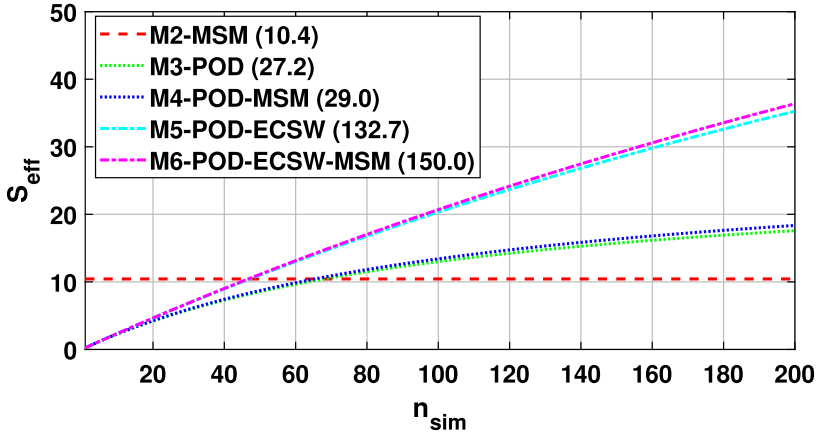

Fig. 15 Effective speedup of MOR for the $54 \mathrm{H} 60$ propeller blade $\left(n_{\psi}=\right.$ 50). The values in the parenthesis are $S$ of each MOR

speedup will remain constant regardless of the number of simulations. In the case of POD-applied MOR, the effective speedup performance will be inferior with the small number of simulations because of the expensive off-line computation. $S_{\text {eff }}$ increases in accordance with the increase of $n_{\text {sim }}$, and approaches the speedup $S$ of each MOR. While $S_{\text {eff }}$ of POD application (M3-POD and M4-POD-MSM) surpasses that of the conventional MSM (M2-MSM) at $n_{\text {sim }}$ of 68 and 66, the hyper-reduction method (M5-POD-ECSW and M6-POD-ECSW-MSM) brings the performance intersection forward to $n_{\text {sim }}$ of 48 and 47 . Despite the increase in the off-line cost due to the sparse NNLS, it is found that the application of the hyper-reduction method will be an ideal choice for the multi-query forced vibration analysis of the rotating component.

\subsection{GT11N turbine blade}

The performance of MOR on a large analysis is validated by its application on the first stage turbine blade of a $75 \mathrm{MW}$ GT11N gas turbine. Some results of the off-line stage, FOM simulation (M1-FOM) and POD application (M3-POD) are obtained from Ref. [6] or the results need to be re-obtained for additional examination. The parameter $\mu=\Omega_{\text {rot }}$ ranges from 1,800 to $5,400 \mathrm{RPM}$ at an interval of $360 \mathrm{RPM}$. The relevant FE discretization is shown in Fig. 16. To describe the complex configuration, the blade will be discretized into millions of DOFs $(N=1,774,512)$ with the corresponding 10-node

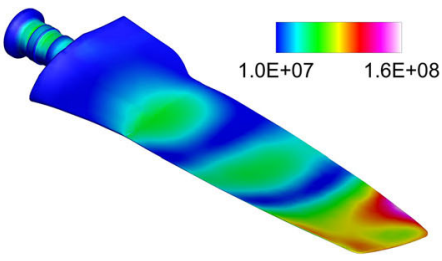

(a)

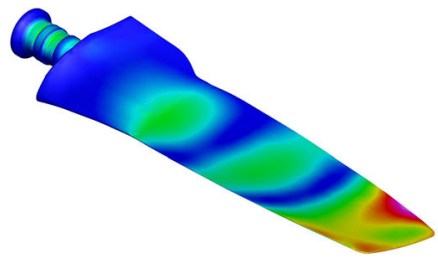

(b)

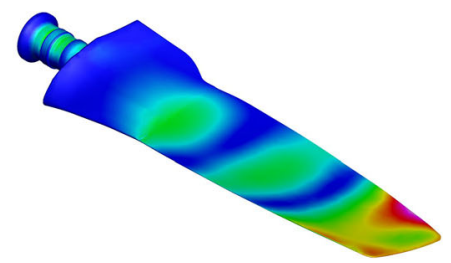

(c)

Fig. 14 von-Mises stress by an alternating stress for 54H60 propeller blade $\left(n_{\psi}=50, \mu=1,530 \mathrm{RPM}, \Omega=250 \mathrm{~Hz}\right)$. All units in Pascal. a M1-FOM, b M5-POD-ECSW, c M6-POD-ECSW-MSM 


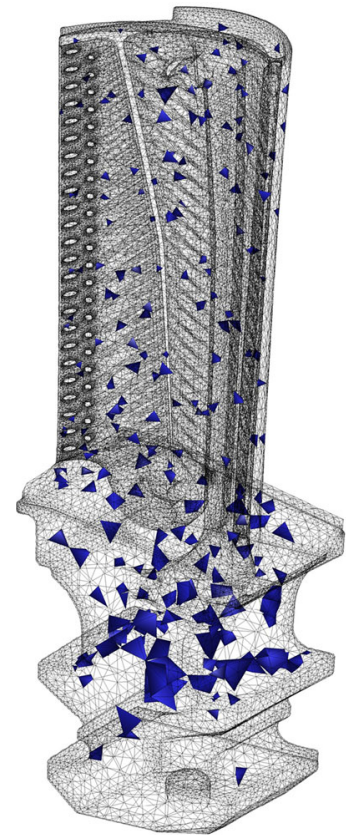

(a)



(b)
Fig. 16 Surface discretization of the GT11N turbine blade and the sampled mesh obtained by the sparse NNLS. a $n_{\psi}=20\left(N_{\tilde{e}}=232\right), \mathbf{b}$ $n_{\psi}=50\left(N_{\tilde{e}}=605\right)$

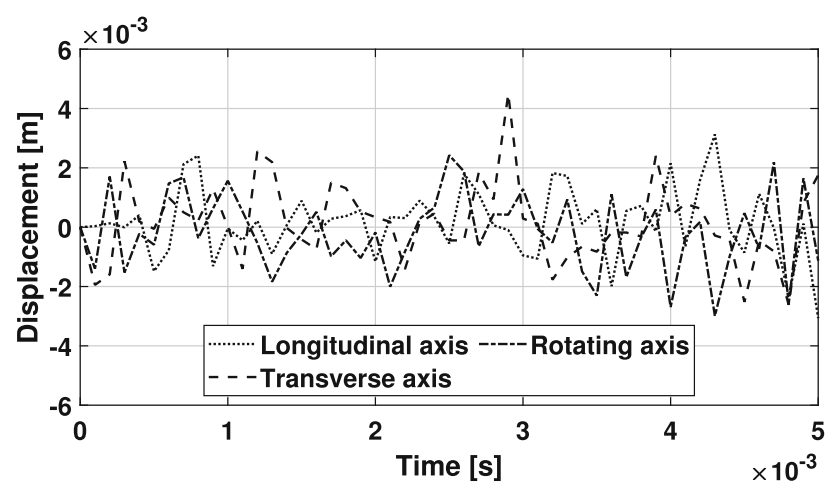

Fig. 17 Time-varying tip displacement of the GT11N turbine blade obtained from the snapshot matrix tetrahedral elements of $N_{e}=376,538$. The material properties of alloy In-738LC are employed [5] with Rayleigh-type viscous damping of mass matrix multiplier of $50 \mathrm{~s}^{-1}$. For the frequency response analysis, a transverse tip harmonic force of $1,000 \mathrm{~N}$ is imposed using the frequency band extending from 500 to $4,500 \mathrm{~Hz}$ at the interval of $40 \mathrm{~Hz}$. The number of eigenmodes is selected as 11 , where the corresponding maximum natural frequency ranges from 8,995 to $9,005 \mathrm{~Hz}$ by the modal analysis (Eq. 8). POD-applied MOR is examined by the various POD bases set of $n_{\psi}$ from 20 to 50 at the interval of 10 .

The snapshot matrix $\mathbf{W}$ is obtained from Ref. [6] and consists of 50 column vectors $\left(N_{s}=50\right)$. For the nonlinear time-transient analysis, the sinusoidal angular velocities of amplitude 3,600 RPM are imposed along the transverse, longitudinal and rotation axes for each. The sinusoidal function oscillates during 5 periods with the frequency of $1,000 \mathrm{~Hz}$. Figure 17 shows the corresponding time-varying structural behavior.

Excluding the computational cost of the sparse NNLS, it takes 20,670.3 seconds for the off-line stage. For the sparse NNLS, $T_{\mathrm{NNLS}}$ is $414,898,1,787$, and 4,152 seconds for each set of POD bases with a tolerance of $\epsilon=0.001$. The sets of the reduced elements $\tilde{\mathbf{E}}$ are shown in Fig. 16 for the cases of the number of POD bases $n_{\psi}=20$ and 50 .

Table 2 summarizes the accuracy and speedup indices $E I_{u}, E I_{\sigma}$, and $S$ for various $n_{\psi}$ in the case of $\mu=3,600$ RPM. Figures 18, 19, 20 show the variations of the performance indices in the parametric axis. Despite the substantial number of DOFs, the accuracy is found to be maintained within $1.5 \%$ and $2.9 \%$ for $E I_{u}$ and $E I_{\sigma}$. In addition, a distinctive speedup is obtained by the hyper-reduction method with a loss of the displacement accuracy $\left(E I_{u}\right)$ of $1.4 \%$ or less. To be more specific, the computational cost of the nonlinear static, modal and frequency response analysis are compared separately in Table. 3. It is found that the remaining computational bottleneck of the nonlinear static analysis is effectively mitigated into single digits by ECSW.

Like Sect. 4.1, the maximum transverse displacement is examined for each frequency location as shown in Figs. 21, 22, 23. Except for the slight deviation in the high frequency region, the frequency response results of MOR are

Table 2 Accuracy and speedup performance of MOR for the GT11N turbine blade ( $\mu=3,600$ RPM)

\begin{tabular}{llll}
\hline MOR framework & $E I_{u}(\%)$ & $E I_{\sigma}(\%)$ & $S$ \\
\hline M2-MSM & 0.17 & 2.52 & 49.4 \\
M3-POD & $8.29 / 0.48 / 0.26 / 0.10$ & $6.60 / 2.35 / 2.06 / 1.78$ & $299.5 / 276.6 / 254.0 / 234.6$ \\
M4-POD-MSM & $8.29 / 0.49 / 0.29 / 0.17$ & $6.63 / 2.70 / 2.62 / 2.53$ & $312.5 / 290.0 / 268.5 / 248.8$ \\
M5-POD-ECSW & $8.45 / 2.95 / 1.41 / 0.68$ & $9.29 / 2.95 / 4.49 / 2.19$ & $1,530.5 / 1,281.3 / 1,097.9 / 936.3$ \\
M6-POD-ECSW-MSM & $8.45 / 3.23 / 1.41 / 0.68$ & $9.31 / 3.23 / 4.77 / 2.86$ & $1,617.3 / 1,437.7 / 1,256.6 / 1,112.0$
\end{tabular}

The values in the multi-entry cells correspond to $n_{\psi}$ of $20,30,40$ and 50 


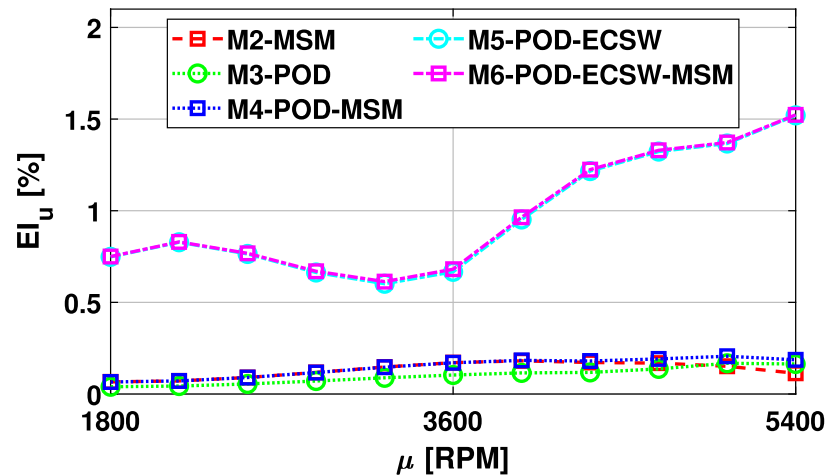

Fig. 18 Accuracy performance of the present MOR for the GT11N turbine blade in terms of $\mu\left(n_{\psi}=50\right): E I_{u}$

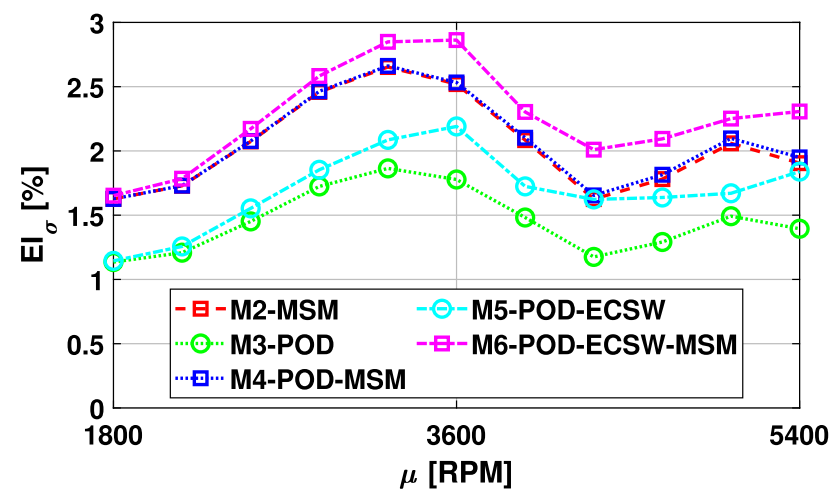

Fig. 19 Accuracy performance of the present MOR for the GT11N turbine blade in terms of $\mu\left(n_{\psi}=50\right): E I_{\sigma}$

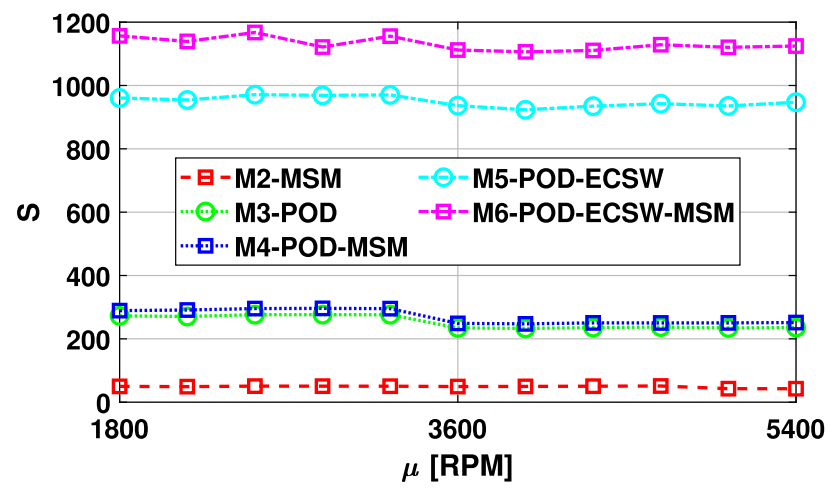

Fig. 20 Speedup performance of the present MOR for the GT11N turbine blade in terms of $\mu\left(n_{\psi}=50\right)$ : $S$

identical to those by FOM. In addition, the maximum vonMises stress by an alternating stress, which is a key factor for HCF investigation, is post-processed as shown in Fig. 24 at the last peak frequency $(4,100 \mathrm{~Hz})$ for the parameter $\mu$ of 5,400 RPM. Despite the large DOFs and complicated configuration, ECSW will provide an accurate stress field when compared with other methods. Figure 25 shows the effective speedup, where POD-applied MOR exceeds the effective speedup of M2-MSM at the range of the number of sim-
Table 3 Average time for the computation $\left(n_{\psi}=50\right)$

\begin{tabular}{llll}
\hline $\begin{array}{l}\text { Analysis } \\
\text { Type }\end{array}$ & $\begin{array}{l}\text { Applied } \\
\text { MOR }\end{array}$ & Equation & $\begin{array}{l}\text { Computational } \\
\text { Time (s) }\end{array}$ \\
\hline Nonlinear static & - & Eq. 2 & 359.0 \\
& POD & Eq. 12 & 89.5 \\
& POD-ECSW & Eq. 20 & 5.5 \\
Modal & - & Eq. 8 & 211.9 \\
& POD & Eq. 14 & 6.4 \\
Frequency response & - & Eq. 7 & $28,213.4$ \\
& MSM & Eq. 9 & 2.5 \\
& POD & Eq. 13 & 13.6
\end{tabular}

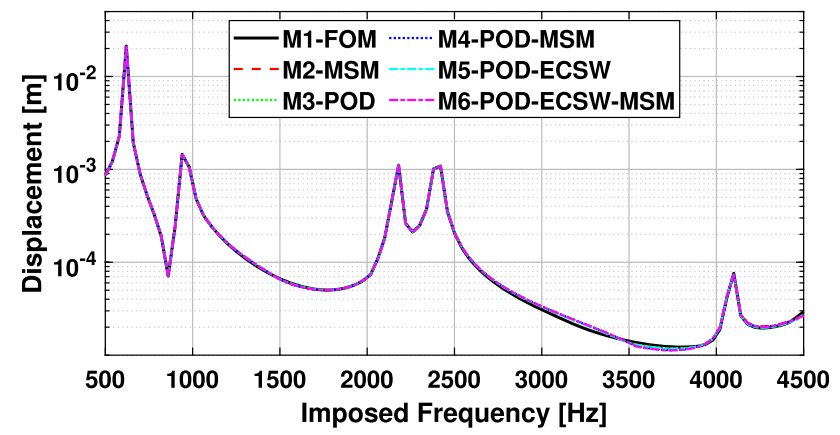

Fig. 21 Maximum transverse displacement amplitude in the GT11N turbine blade $\left(n_{\psi}=50\right): \mu=1,800 \mathrm{RPM}$

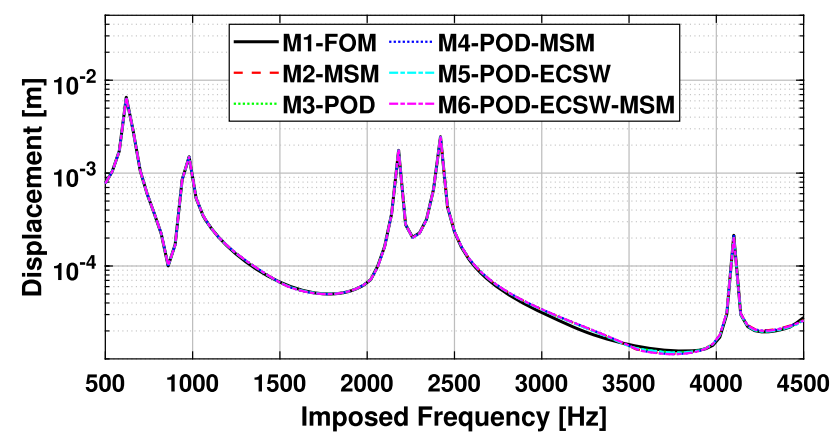

Fig. 22 Maximum transverse displacement amplitude in the GT11N turbine blade $\left(n_{\psi}=50\right): \mu=3,600 \mathrm{RPM}$

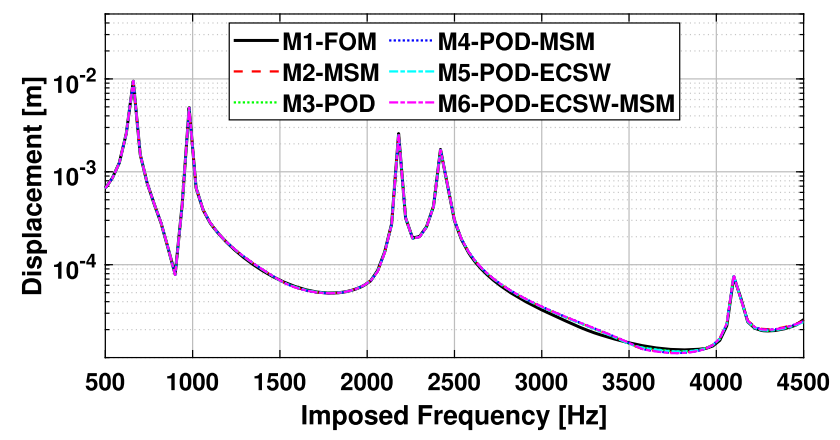

Fig. 23 Maximum transverse displacement amplitude in the GT11N turbine blade $\left(n_{\psi}=50\right): \mu=5,400 \mathrm{RPM}$ 
(a)





(b)

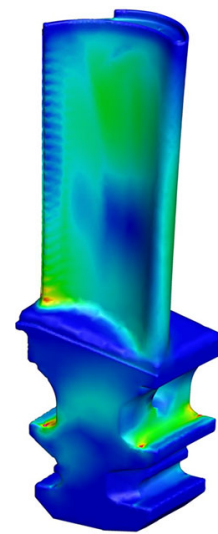

(c)
Fig. 24 von-Mises stress by an alternating stress for the GT11N turbine blade $\left(n_{\psi}=50, \mu=5,400 \mathrm{RPM}, \Omega=4,100 \mathrm{~Hz}\right)$. All units in Pascal. a M1-FOM, b M5-POD-ECSW, c M6-POD-ECSW-MSM

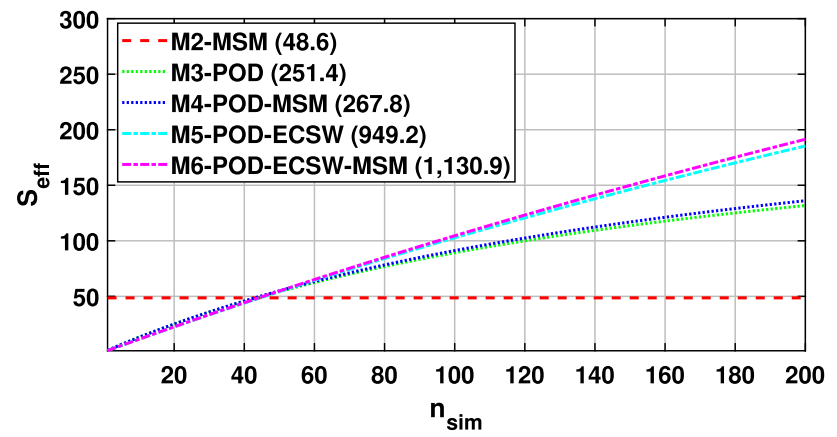

Fig. 25 Effective speedup performance of MOR for the GT11N turbine blade $\left(n_{\psi}=50\right)$. The values in the parenthesis are $S$ of each MOR

ulations from 43 to 45 . Despite the improved speedup $S$, ECSW-applied MOR shows similar effective speedup performance $S_{\text {eff }}$ as that without the hyper-reduction method in the range of $n_{\text {sim }}<55$, because of the increased cost of the sparse NNLS. In practice, it will be proper to employ the parallel computing techniques for such large DOF off-line including the sparse NNLS, as shown in Ref. [14]. Thereafter, ECSW-applied MOR will appear to be superior $S_{\text {eff }}$ to the other methods. By observing the accuracy and speedup performance shown above, the proposed hyper-reduction application (M5-POD-ECSW and M6-POD-ECSW-MSM) will be appropriate for large forced vibration analysis.

\section{Conclusions}

The qualitative and quantitative investigation is performed for MOR application on the rotating component forced vibration analysis. The computational procedure of the forced vibration analysis includes the nonlinear static analysis for the pre-stressed stiffness and the linear frequency response analysis for the harmonic displacement. Based on MOR frameworks by MSM and POD, the hyper-reduction method (ECSW) is newly applied to relieve the remaining computational bottleneck in the iterative nonlinear analysis. Two practical applications, the slender propeller blade and complicated turbine blade, are selected as the rotating component examples to validate the present MOR performance in the environment of the high geometrical nonlinearity and the large DOFs. Through the studies, the following conclusions are derived:

- Regardless of the parameter (rotating speed) variation, the hyper-reduction method provides speedup performance which is ten to twenty times superior than the conventional MSM (M2-MSM), with admissible accuracy loss.

- In terms of the effective speedup including off-line costs in consideration, the application of ECSW shows its advantage for multi-query simulations.

- For the large number of DOFs, the ECSW-applied MOR exhibits a rather deteriorated effective speedup performance for the small number of simulations due to the increased cost of the sparse NNLS. Nevertheless, an exponentially increased speedup is observed for the large number of simulations.

- All present MOR frameworks provide precise alternating stress distribution, which validates the employment of the hyper-reduction method on the rotating component forced vibration analysis.

In the future, the present MOR applications will extend to the material nonlinearity of viscoplasticity. Thus, the applicability of the present MOR on a low cycle fatigue analysis will be examined.

Acknowledgements This work was supported by the UAV HighEfficiency Turbine Research Center program of the Defense Acquisition Program Administration and Agency for Defense Development, Republic of Korea. In addition, we thank the Republic of Korea Air Force Aero Technology Research Institute for providing the three-dimensional CAD drawing of the $54 \mathrm{H} 60$ propeller blade.

\section{Declarations}

Conflict of interest The authors declare that they have no conflict of interest.

Open Access This article is licensed under a Creative Commons Attribution 4.0 International License, which permits use, sharing, adaptation, distribution and reproduction in any medium or format, as long as you give appropriate credit to the original author(s) and the source, provide a link to the Creative Commons licence, and indicate if changes were made. The images or other third party material in this article are included in the article's Creative Commons licence, unless indicated otherwise in a credit line to the material. If material 
is not included in the article's Creative Commons licence and your intended use is not permitted by statutory regulation or exceeds the permitted use, you will need to obtain permission directly from the copyright holder. To view a copy of this licence, visit http://creativecomm ons.org/licenses/by/4.0/.

\section{References}

1. Misra AK, Greenbauer-Seng LA (2013) Aerospace propulsion and power materials and structures research at NASA Glenn research center. J Aerosp Eng 26(2):459-490. https://doi.org/10. 1061/(ASCE)AS.1943-5525.0000325

2. Ewins DJ (1998) Basic structural dynamics. In: Platzer MF, Carta FO (eds) AGARD manual on aeroelasticity in axial-flow turbomachines-Volume 2: structural dynamics and aeroelasticity. North Atlantic Treaty Organization (NATO), Neuilly sur Seine, pp $31-46$

3. Bathe KJ (2014) Finite element procedures. Klaus-Jürgen Bathe, Watertown

4. Guo D, Chu FL, Zheng ZC (2001) The influence of rotation on vibration of a thick cylindrical shell. J Sound Vib 242(3):487-505. https://doi.org/10.1006/jsvi.2000.3356

5. Kim Y, Cho H, Park S, Kim H, Shin SJ (2018) Advanced structural analysis based on reduced order modeling for gas turbine blade. AIAA J 56(8):3369-3373. https://doi.org/10.2514/1.J057063

6. Kang S, Kim Y, Song D, Cho H, Shin SJ (2022) Numerical characterization of turbine blade dynamic properties via proper orthogonal decomposition. J Phys Conf Ser (in press)

7. Kim Y, Kang S, Cho H, Shin SJ (2021) Improved nonlinear analysis of a propeller blade based on hyper-reduction. AIAA J. https://doi. org/10.2514/1.J060742

8. Chatterjee A (2000) An introduction to the proper orthogonal decomposition. Curr Sci 78(7):808-817

9. Rowley CW (2005) Model reduction for fluids, using balanced proper orthogonal decomposition. Int J Bifurc Chaos Appl Sci Eng 15(3):997-1013. https://doi.org/10.1142/S0218127405012429

10. Thomas PV, ElSayed MSA, Walch D (2019) Review of model order reduction methods and their applications in aeroelasticity loads analysis for design optimization of complex airframes. J Aerosp Eng 32(2):1-19. https://doi.org/10.1061/(ASCE)AS.1943-5525. 0000972

11. Ri JS, Hong HS (2019) A basis reduction method using proper orthogonal decomposition for shakedown analysis of kinematic hardening material. Comput Mech 64:1-13. https://doi.org/10. 1007/s00466-018-1653-y

12. Chaturantabut S, Sorensen DC (2010) Nonlinear model reduction via discrete empirical interpolation. SIAM J Sci Comput 32(5):2737-2764. https://doi.org/10.1137/090766498
13. Carlberg C, Farhat C, Cortial J, Amsallem D (2013) The GNAT method for nonlinear model reduction: effective implementation and application to computational fluid dynamics and turbulent flows. J Comput Phys 242:623-647. https://doi.org/10.1016/j.jcp. 2013.02.028

14. Farhat C, Avery P, Chapman T, Cortial J (2014) Dimensional reduction of nonlinear finite element dynamic models with finite rotations and energy-based mesh sampling and weighting for computational efficiency. Int J Numer Methods Eng 98(9):652-662. https://doi.org/10.1002/nme.4668

15. Cho H, Shin SJ, Kim H, Cho M (2020) Enhanced model-order reduction approach via online adaptation for parametrized nonlinear structural problems. Comput Mech 65:331-353. https://doi. org/10.1007/s00466-019-01771-7

16. Anonymous (2018) ANSYS Mechanical user's guide; release 19.2. ANSYS Inc., Canonsburg

17. Anonymous (2019) Intel Math Kernel Library developer reference; release 2020. Intel, Santa Clara

18. Anonymous (2018) ANSYS meshing user's guide; release 19.2. ANSYS Inc., Canonsburg

19. Cardarelli F (2008) Materials handbook: a concise desktop reference, 2nd edn. Springer, London

20. Lawson CL, Hanson RJ (1987) Solving least squares problems. Society for Industrial and Applied Mathematics (SIAM), Philadelphia

21. Lehoucq RB, Sorensen DC, Yang C (1998) ARPACK users' guide: solution of large scale eigenvalue problems with implicitly restarted Arnoldi methods. Society for Industrial and Applied Mathematics (SIAM), Philadelphia

Publisher's Note Springer Nature remains neutral with regard to jurisdictional claims in published maps and institutional affiliations. 